\title{
Error bounds for the spectral approximation of the potential of a homogeneous almost spherical body
}

\author{
BLAŽEJ BuCHA $^{1}$, LORENZO RossI ${ }^{2 !}$ AND FERNANDO SANSÒ $^{2}$ \\ 1 Department of Theoretical Geodesy and Geoinformatics, Slovak University of Technology, \\ Radlinského 11, 81005 Bratislava, Slovakia (blazej.bucha@stuba.sk) \\ 2 Department of Civil and Environmental Engineering, Politecnico di Milano, Piazza Leonardo \\ da Vinci 32, 20133 Milan, Italy (lorenzo1.rossi@polimi.it, fernando.sanso@polimi.it)
}

Received: April 20, 2021; Revised: July 2, 2021; Accepted: July 20, 2021

\begin{abstract}
Several kinds of approximation of the gravitational potential of a homogeneous body by truncated spherical harmonics series are in use in physical geodesy. However, only one of them is capable of a representation converging to the true potential in the whole layer between the Brillouin sphere and the Bjerhammar sphere of the body. We aim at providing various majorizations, namely upper bounds, of the error with the double purpose of proving explicitly the convergence in the sense of different norms and of giving computable bounds, that might be used in numerical studies. The first aim is reached for all the norms. For the second, however, it turns out that among the bounds, when applied to the example of the terrain correction of the Earth, only those referring to the mean absolute error and the mean squared error at the level of Brillouin sphere of minimum radius give significant and useful results. In order to make the computation an easy exercise, a simple approximate formula has been developed requiring only the use of the distribution function of the heights of the surface of the body with respect to the Bjerhammar sphere.
\end{abstract}

Keywords: homogeneous bodies, gravity field, spectral approximation, convergence theory, numerical bounds

\section{INTRODUCTION}

The gravitational potential $u(\boldsymbol{x})$ of a homogeneous body $B$ can be expressed as the Newton integral over $B$ :

$$
u(\boldsymbol{x})=\mu \int_{B} \frac{1}{\ell_{x y}} d \boldsymbol{y}
$$




\section{B. Bucha et al.}

where $\mu=G \rho, G$ is the Newton constant, $\rho$ is the mass density, $\ell_{x y}=|\boldsymbol{x}-\boldsymbol{y}|, \boldsymbol{x}$ is the computational point, and $\boldsymbol{y}$ is the running point. Equation (1) is valid for every $\boldsymbol{x}$ in $\mathbb{R}^{3}$ space.

However, when $B$ has approximately a spherical shape, but with an irregular almost spherical boundary, it is often convenient to use a spherical harmonic expansion of Netwon kernel, which then has to be truncated to some maximum degree $N$, in order to provide practical formulas, useful for gravity calculations. In this way a spectral approximation $u_{N}(\boldsymbol{x})$ of $u(\boldsymbol{x})$ is created, also called a global gravity model with maximum degree $N$. Therefore, the corresponding error is:

$$
\varepsilon_{N}(\boldsymbol{x})=u(\boldsymbol{x})-u_{N}(\boldsymbol{x}) .
$$

The purpose of the present study is to provide a tight upper bound to $\varepsilon_{N}(\boldsymbol{x})$ both in a point-wise sense and in a mean sense, as it will be defined. The bounds will be tight because derived from functional inequalities rather than averaging over realizations of $u(\boldsymbol{x})$ considered as a random field, as it is done for instance in collocation theory. We notice that with the above specification, the error $\varepsilon_{N}(\boldsymbol{x})$ is just an omission error and no noise is assumed to be present in the data.

The item is traditional in geodesy where for instance the body $B$ can be considered as that of the topographic masses, floating on a sphere of radius $R_{0}$ (spherical approximation) when we want to compute a global model of its gravity effects (Moritz, 1980; Balmino, 1994; Wieczorek, 2015; Freeden and Schreiner, 2009; Sansò and Sideris, 2012; Balmino et al., 2012; Hirt and Kuhn, 2014; Hirt et al., 2016, 2019; Jekeli, 2017; Górski et al., 2018).

The problem has been revised by the need of accurately representing the gravity field of irregular celestial bodies, like asteroids, e.g. for the purpose of manoeuvring a spacecraft close to their surface (Balmino, 1994; Takahashi and Scheeres, 2014; Reimond and Baur, 2016; Górski et al., 2018). In this respect, during the discussion on the results reported in Bucha and Sanso (2021), the authors have also tried to examine analytically the problem of providing bounds to the error of spectral approximation of the true potential, obtaining the material that we decided to collect in a separate paper.

In Bucha and Sansò (2021), three possible methods to construct a spectral approximation of the potential $u(\boldsymbol{x})$ were discussed.

A) Expansion of $\ell_{x y}^{-1}$ into a series of external solid harmonics as a function of $\boldsymbol{x}$ and then truncate it at a maximum degree $N$; the corresponding Newton integral gives the spectral approximation $u_{N}^{e}(\boldsymbol{x})$ of $u(\boldsymbol{x})$. The coefficients $u_{n m}^{e}$ of the truncated series are then usually expressed as functionals of the shape of the boundary $S$ of $B$ (Balmino, 1994; Bucha et al., 2019). This approach has been called the spectral forward gravity modelling in Bucha and Sansò (2021). 
B) Expansion of $\ell_{x y}^{-1}$ into a series of solid spherical harmonics, yet with two alternative expansions; one internal, when $\boldsymbol{x}$ is closer to the origin than the integration point $\boldsymbol{y}$, the other external, in the opposite case. The calculation of the Newton integral for the above series, truncated at maximum degree $N$, yields the spectral approximation $u_{N}(\boldsymbol{x})$; the method has been called internal-external development in Bucha and Sansò (2021).

C) The spectral approximation $\hat{u}_{N}^{l s}(\boldsymbol{x})$ as a linear combination of external solid spherical harmonics, choosing the coefficients such that the $L_{2}$ norm of the difference $u(\boldsymbol{x})-\hat{u}_{N}^{l s}(\boldsymbol{x})$ is minimized on the surface $S$ of the body $B$.

Each method produces a different error function $\varepsilon_{N}(\boldsymbol{x})$ which should be separately analysed.

Method A, although very common among geodesists, suffers of one fundamental drawback; although the integral of the external series of $\ell_{x y}^{-1}$ is known to converge for $\boldsymbol{x}$ outside a sphere encompassing all the masses (i.e. the Brillouin sphere), it is also known that its convergence inside the minimum Brillouin radius is a very unstable and questionable property (see Moritz, 1980; Sansò and Sideris, 2012), which has started to appear also numerically (Hirt and Kuhn, 2017) when the maximum degree is pushed towards high figures (e.g. of the order of $10^{4}$ for the Earth). In any event, this item has a long history in geodetic literature, for which we mention only the works by Jekeli (1983), Sjöberg (1977, 1999), Agren (2004). For this reason we will not work on Method A in the paper, although more precise quantification of its error remains an interesting question.

Method B, contrary to Method A, is based on an exact computation of the integral of the spectral approximation to $\ell_{x y}^{-1}$ over the body $B$, even when $\boldsymbol{x}$ is below the minimum Brillouin sphere. This has been understood since a long time and presented in geodetic literature, e.g. in Hotine (1969); Sjöberg (1977); Martinec (1998); Górski et al. (2018). The approach is not very efficient on a numerical ground, especially when computational points are located on the irregular body surface, as shown in Bucha and Sansò (2021), yet it is stable and everywhere convergent, as one would expect and we will show.

The Method C is numerically efficient (Bucha and Sansò, 2021) and well understood from the mathematical point of view. In fact, it is known (Sansò and Sideris, 2012, Chapter 13,4-13,5) that the least squares estimate, $\hat{u}_{N}^{l s}(\boldsymbol{x})$, approaches the true potential $u(\boldsymbol{x})$ in the $L_{2}$ sense on $S$ and uniformly on any compact $K$ external to $S$, on condition that $\left.u(\boldsymbol{x})\right|_{S}$ is a function of $L_{2}(S)$. The only warning in using $\hat{u}_{N}^{l s}(\boldsymbol{x})$ is that in general it does not approximate $u(\boldsymbol{x})$ inside the masses and its harmonic coefficients, $\hat{u}_{n m}^{l s}(N)$, change with $N$, converging when $N \rightarrow \infty$ to the coefficients of the external series, $u_{n m}^{e}$ (Sansò and Sideris, 2012). In other words, for a fixed $N$ they cannot be confused with the 
coefficients estimated from satellite observations or with the ones computed by Method A. Moreover, by its very nature, the error of the least squares solution is smaller than that of Method B, so any majorization of the latter will be valid for the former too.

For the above reasons in this paper we will focus essentially on Method B.

The paper is organized as follows: in Section 2 we introduce the chosen notation, in Sections 3-5 we derive different majorizations of $\varepsilon_{N}(\boldsymbol{x})$; in Section 6 the above formulas are used to get numerical estimates of the error for the case of Earth terrain effect; conclusions follow.

\section{NOTATION, HYPOTHESES AND DEFINITION OF THE PROBLEM}

In this paper we shall assume that the body $B$ is the support of a mass distribution with constant density $\rho$ and has a starshaped boundary, the surface $S$.

In the paper the following notation will be used (see Fig. 1):

$B$ : body with radius $r=R(\vartheta, \lambda)=R_{\sigma}$, where $R_{0} \leq R_{\sigma} \leq \bar{R}$;

$B_{0}$ : Bjerhammar sphere;

$\bar{B}$ : Brillouin sphere, i.e. the sphere with radius $r=\bar{R}(\forall \vartheta, \lambda)$;

$\sigma=(\vartheta, \lambda):$ angular spherical coordinates;

$\boldsymbol{x}=\left(r, \sigma_{x}\right)$ : computational point;

$\boldsymbol{y}=\left(s, \sigma_{y}\right)$ : running point (for the integral);

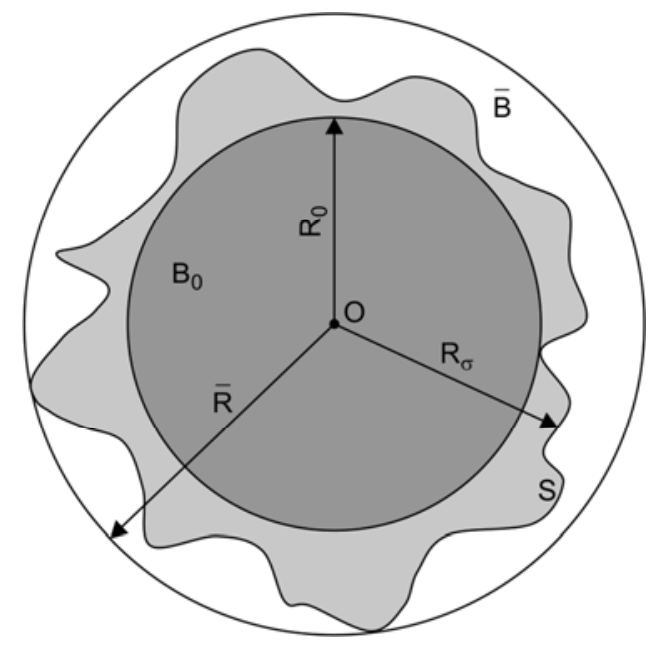

Fig. 1. Notation used in the paper. $B_{0}$ : Bjerhammar sphere with radius $R_{0} \leq \min _{\sigma} R_{\sigma} ; \bar{B}$ : Brillouin sphere, i.e. the sphere with radius $r=\bar{R} \geq \max _{\sigma} R_{\sigma} ; S$ : boundary of the body $B$ with radius $r=R(\vartheta, \lambda)=R_{\sigma}$, where $R_{0} \leq R_{\sigma} \leq \bar{R} ; \sigma=(\vartheta, \lambda)$ : angular spherical coordinates. 


\section{Approximation of the potential of a homogeneous almost spherical body}

$S=\left\{r=R_{\sigma}\right\}:$ boundary of $B$;

$R_{0} \leq \min _{\sigma} R_{\sigma}:$ Bjerhammar radius (radius of $B_{0}$ );

$\bar{R} \geq \max _{\sigma} R_{\sigma}$ : Brillouin radius (radius of $\bar{B}$ );

$\mu=G \rho$ : the product of Newton constant $G$ and the constant density of the body $\rho$;

$H_{\sigma}=R_{\sigma}-R_{0}$ : the "height" of a point lying on $S\left(R_{\sigma}, \sigma\right)$;

$\bar{H}=\max _{\sigma} H_{\sigma}$;

$\delta B=B \backslash B_{0}=\left\{R_{0} \leq r \leq R_{\sigma}\right\}$

$\ell_{x y}=|\boldsymbol{x}-\boldsymbol{y}|:$ distance between the points $\boldsymbol{x}$ and $\boldsymbol{y}$;

$\psi_{x y}=\psi_{\sigma_{x} \sigma_{y}}:$ angular distance between the vectors of points $\boldsymbol{x}$ and $\boldsymbol{y}$;

$H(t)$ : Heaviside step function $H(t)=\left\{\begin{array}{cl}1 & t>0, \\ 1 / 2 & t=0, \\ 0 & t<0 .\end{array}\right.$

In the rest of the paper, we will choose $\bar{R}$ as the minimum Brilluoin radius and $R_{0}$ as the maximum Bjerhammar radius, so that we have $\bar{H}=\bar{R}-R_{0}$, too. Let us also notice that the difference between the Heaviside function $H(t)$ and the height $H_{\sigma}$ will always be clear from the context. In any event, the symbols should not be confused also observing that the Heaviside function is always written with its argument in parenthesis, $H(t)$, while heights are always without further specifications or with an index like $H_{\sigma}$. We will call $\sigma$ and $\mathrm{d} \sigma$ the unit sphere and its area element, respectively, when no confusion can arise with the coordinates of a single point. Moreover, we will make use of the well known formulas:

$$
P_{n}\left(\cos \psi_{x y}\right)=\frac{1}{2 n+1} \sum_{m=-n}^{n} Y_{n m}\left(\sigma_{x}\right) Y_{n m}\left(\sigma_{y}\right),
$$

where

$$
Y_{n m}\left(\sigma_{x}\right)=\bar{P}_{n m}\left(\cos \vartheta_{x}\right) f_{m}\left(\lambda_{x}\right), \text { and } f_{m}\left(\lambda_{x}\right)=\left\{\begin{array}{ccc}
\cos m \lambda_{x} & \text { for } & m \geq 0, \\
\sin |m| \lambda_{x} & \text { for } & m<0,
\end{array}\right.
$$

also called summation formula, and

$$
\begin{gathered}
\ell_{x y}^{-1}=\left(r^{2}+s^{2}-2 r s \cos \psi_{x y}\right)^{-1 / 2}=\sum_{n=0}^{\infty} F_{n}(r, s) P_{n}\left(\cos \psi_{x y}\right), \\
F_{n}(r, s)=\frac{s^{n}}{r^{n+1}} H(r-s)+\frac{r^{n}}{s^{n+1}} H(s-r),
\end{gathered}
$$




\section{B. Bucha et al.}

where $\ell_{x y}^{-1}$ is concisely expanded in the form of external and internal series expansion according whether $r \geq s$ or $r<s$.

Finally we will call $\ell_{N, x y}$ the series of Eq. (4) truncated to the maximum degree $N$, namely:

$$
\ell_{N, x y}^{-1}=\sum_{n=0}^{N} F_{n}(r, s) P_{n}\left(\cos \psi_{x y}\right) .
$$

With the above notation, the gravitational potential of $B$ is given by:

$$
u(\boldsymbol{x})=\mu \int_{B} \frac{1}{\ell_{x y}} d \boldsymbol{y}
$$

while its internal-external spectral approximation is:

$$
u_{N}(\boldsymbol{x})=\mu \int_{B} \frac{1}{\ell_{N, x y}} d \boldsymbol{y},
$$

with $\boldsymbol{x}$ being any point in $\mathbb{R}^{3}$. The corresponding internal-external error $\varepsilon_{N}(\boldsymbol{x})$ is then

$$
\varepsilon_{N}(\boldsymbol{x})=\mu \int_{B}\left(\frac{1}{\ell_{x y}}-\frac{1}{\ell_{N, x y}}\right) d \boldsymbol{y}=\mu \int_{B} \sum_{n=N+1}^{\infty} F_{n}(r, s) P_{n}\left(\cos \psi_{x y}\right) d \boldsymbol{y} .
$$

A first significant simplification of Eq. (9) is given by the following remark; let us split $B$ into $B=B_{0} \cup \delta B$ and write Eq. (9) as

$$
\begin{gathered}
\varepsilon_{N}(\boldsymbol{x})=\mu \int_{B_{0}} \sum_{n=N+1}^{\infty} F_{n}(r, s) P_{n}\left(\cos \psi_{x y}\right) d \boldsymbol{y} \\
+\mu \int_{\delta B} \sum_{n=N+1}^{\infty} F_{n}(r, s) P_{n}\left(\cos \psi_{x y}\right) d \boldsymbol{y} .
\end{gathered}
$$

Since

$$
\begin{gathered}
\sum_{n=N+1}^{\infty} \int_{B_{0}} F_{n}(r, s) P_{n}\left(\cos \psi_{x y}\right) d \boldsymbol{y} \\
=\sum_{n=N+1}^{\infty} \int_{0}^{R_{0}} F_{n}(r, s) s^{2} \mathrm{~d} s \int_{\sigma} P_{n}\left(\cos \psi_{x y}\right) d \sigma_{y}=0,
\end{gathered}
$$

because $P_{n}\left(\cos \psi_{x y}\right)$ has 0 mean on the unit sphere $\forall n \geq 1$, we see that Eq. (11) corresponds to the first integral on the right-hand side of Eq. (10). Therefore it disappears and we are left with 


$$
\begin{gathered}
\varepsilon_{N}(\boldsymbol{x})=\mu \int_{\delta B}\left(\frac{1}{\ell_{x y}}-\frac{1}{\ell_{N, x y}}\right) d \boldsymbol{y} \\
=\mu \sum_{n=N+1}^{\infty} \int_{\sigma} d \sigma_{y}\left[\int_{R_{0}}^{R_{\sigma}} F_{n}(r, s) s^{2} \mathrm{~d} s\right] P_{n}\left(\cos \psi_{x y}\right) .
\end{gathered}
$$

In the following, starting from Eq. (12), we will derive several different majorizations, namely upper bounds, of the error $\varepsilon_{N}(\boldsymbol{x})$.

Remark. Before doing the majorization job, we would like to make few comments on the hypothesis that the density in $B$ should be constant. Is this hypothesis necessary to derive Eq. (12)? This is not the case, in fact if $\rho$ is variable with the integration point $y$, we could still have

$$
\int_{B_{0}} \rho(\boldsymbol{y}) \sum_{n=N+1}^{\infty} F_{n}(r, s) P_{n}\left(\cos \psi_{x y}\right) d \boldsymbol{y}=0,
$$

if $\rho(y)$ could be expressed in the form

$$
\rho(\boldsymbol{y})=\sum_{n=0}^{N} \sum_{m=-n}^{n} \rho_{n m}(s) Y_{n m}\left(\sigma_{y}\right) .
$$

This includes quite realistic layered models, with a lateral variation that can be developed in surface spherical harmonics up to degree $N$.

In addition, if the density $\rho$ is variable, but with a finite upper bound $\bar{\rho}$, inside $\delta B$ (i.e. $R_{0} \leq s \leq R_{\sigma}$ ), on condition of redefining $\mu$ as $\bar{\mu}=G \bar{\rho}$, Eq. (12) becomes

$$
\left|\varepsilon_{N}(\boldsymbol{x})\right|=G \int_{\delta B}\left|\frac{1}{\ell_{x y}}-\frac{1}{\ell_{N, x y}}\right| \rho(\boldsymbol{y}) d \boldsymbol{y} \leq \bar{\mu} \int_{\delta B}\left|\frac{1}{\ell_{x y}}-\frac{1}{\ell_{N, x y}}\right| d \boldsymbol{y},
$$

and all the derivations we will make in the next section still hold equally well. Due to Eq. (15) and for the sake of notation simplicity, we will refer to both $\mu$ and $\bar{\mu}$ simply as $\mu$ hereafter.

\section{THE MAJORIZATION IN $C\left(\bar{B} \backslash B_{0}\right)$}

We aim at finding a majorization for

$$
\left\|\varepsilon_{N}\right\|_{C(\bar{B})}=\sup _{\boldsymbol{x} \in \bar{B} \backslash B_{0}}\left|\varepsilon_{N}(\boldsymbol{x})\right|,
$$




\section{B. Bucha et al.}

i.e. majorizing $\left|\varepsilon_{N}(\boldsymbol{x})\right|$ for $R_{0} \leq r \leq \bar{R}$. We will use the relation (see Sansò and Sideris, 2012, Chapter 3.4)

$$
\int_{\sigma} P_{j}\left(\cos \psi_{z y}\right) P_{n}\left(\cos \psi_{x y}\right) d \sigma_{y}=\frac{4 \pi}{2 n+1} P_{n}\left(\cos \psi_{z x}\right) \delta_{n j},
$$

that can be easily derived from Eq. (3) considering the orthonormality of $\left\{Y_{n m}(\sigma)\right\}$ in $L_{2}(\sigma)$.

It also follows from Eq. (17) that if

$$
f\left(\sigma_{y}\right)=\sum_{n=0}^{\infty} f_{n} P_{n}\left(\cos \psi_{x y}\right)
$$

then

$$
\int_{\sigma} f\left(\sigma_{y}\right)^{2} d \sigma_{y}=4 \pi \sum_{n=0}^{\infty} \frac{f_{n}^{2}}{2 n+1} .
$$

To get our result, we firstly apply the Schwarz inequality to Eq. (12), getting for $\boldsymbol{x} \in \bar{B} \backslash B_{0}$

$$
\left|\varepsilon_{N}(\boldsymbol{x})\right|^{2} \leq \mu^{2} m(\delta B) \int_{\delta B}\left(\frac{1}{\ell_{x y}}-\frac{1}{\ell_{N, x y}}\right)^{2} d \boldsymbol{y}
$$

where $m(\delta B)$ is the measure (volume) of $\delta B$. Equation (20) is justified because $\left(1 / \ell_{x y}-1 / \ell_{N, x y}\right)^{2}$ is in $L_{2}(\delta B)$, see Appendix A. Then we majorize the integral of the above positive function on $\delta B$ with that on the larger set $\bar{B} \backslash B_{0}$ and we further apply Eq. (19), obtaining

$$
\begin{gathered}
\left|\varepsilon_{N}(\boldsymbol{x})\right|^{2} \leq \mu^{2} m(\delta B) \int_{R_{0}}^{\bar{R}} \mathrm{~d} s s^{2} \int_{\sigma} d \sigma\left(\sum_{n=N+1}^{\infty} F_{n}(r, s) P_{n}\left(\cos \psi_{x y}\right)\right)^{2} \\
=\mu^{2} m(\delta B) \int_{R_{0}}^{\bar{R}} \mathrm{~d} s s^{2} 4 \pi \sum_{n=N+1}^{\infty} \frac{F_{n}^{2}(r, s)}{2 n+1} .
\end{gathered}
$$

The elementary calculation of the radial integral of $F_{n}^{2}(r, s)$ is performed in Appendix B, where we put, see Eq. (B2),

$$
(R \leq \bar{R}) \quad H_{n}(r, R)=\int_{R_{0}}^{R} F_{n}^{2}(r, s) s^{2} \mathrm{~d} s .
$$


We will use Eq. (B15), and the definition of $a_{n}$ therein, in Eq. (21), writing

$$
\begin{aligned}
& \frac{H_{n}(r, \bar{R})}{2 n+1}=\int_{R_{0}}^{\bar{R}} \mathrm{~d} s s^{2} \frac{F_{n}^{2}(r, s)}{2 n+1} \leq \frac{a_{n}}{2 n+1} \bar{R} \\
& =\frac{2 \bar{R}}{(2 n+3)(2 n-1)}=\frac{\bar{R}}{2}\left(\frac{1}{2 n-1}-\frac{1}{2 n+3}\right) .
\end{aligned}
$$

Therefore

$$
\sum_{n=N+1}^{\infty} \frac{H_{n}(r, \bar{R})}{2 n+1} \leq \frac{\bar{R}}{2}\left(\frac{1}{2 N+1}+\frac{1}{2 N+3}\right) \cong \frac{\bar{R}}{2(N+1)}
$$

so that Eq. (21) becomes

$$
\left|\varepsilon_{N}(\boldsymbol{x})\right| \leq \mu \sqrt{\frac{4 \pi m(\delta B) \bar{R}}{2(N+1)}}=C_{N}
$$

Three considerations are in order before going to other inequalities. The first is that Eq. (25) proves the uniform convergence of $u_{N}(\boldsymbol{x})$ to $u(\boldsymbol{x})$ in the "topographic layer", $\bar{B} \backslash B_{0}$; with some extra effort it is not difficult to prove that in reality such a convergence holds for the whole $\mathbb{R}^{3}$. The second comment is that the convergence of $C_{N}$ to zero for $N \rightarrow \infty$, from Eq. (25), is very slow. The third remark is that the procedure applied to derive Eq. (25) has been very rough, specially because we have, as a matter of fact, filled the whole layer $\bar{B} \backslash B_{0}$ with a mass having a constant density $\rho$, in order to switch from Eq. (20) to Eq. (21). Therefore we can expect Eq. (25) to be quite pessimistic, as it is confirmed by Example 1 in Section 6, where we will apply Eq. (25) to estimate the error for the global terrain effect of the Earth and we find a bound of $\sim 50 \mathrm{~m}$ for $N=10000$ in terms of the corresponding height anomaly, certainly not a very useful figure.

\section{THE MAJORIZATION OF THE MEAN ABSOLUTE ERROR IN THE LAYER $R_{0} \leq r \leq \bar{R}$}

We define the absolute mean value of $\varepsilon_{N}(\boldsymbol{x})=\varepsilon_{N}\left(r, \sigma_{x}\right)$ over a sphere of radius $r: R_{0} \leq r \leq \bar{R}$ as

$$
M_{N}(r)=\frac{1}{4 \pi} \iint_{\sigma}\left|\varepsilon_{N}\left(r, \sigma_{x}\right)\right| d \sigma_{x}
$$

Going back to Eq. (12) and using the definition of $G_{n}(r, R)$, see also Eq. (B2), 


$$
G_{n}(r, R)=\int_{R_{0}}^{R} F_{n}(r, s) s^{2} \mathrm{~d} s
$$

we can write

$$
\left|\varepsilon_{N}(\boldsymbol{x})\right| \leq \mu \sum_{n=N+1}^{\infty} \int_{\sigma} d \sigma_{y} G_{n}\left(r, R_{\sigma_{y}}\right)\left|P_{n}\left(\cos \psi_{x y}\right)\right|,
$$

and

$$
M_{N}(r) \leq \mu \sum_{n=N+1}^{\infty} \int_{\sigma} d \sigma_{y} G_{n}\left(r, R_{\sigma_{y}}\right) \frac{1}{4 \pi} \int_{\sigma}\left|P_{n}\left(\cos \psi_{x y}\right)\right| d \sigma_{x} .
$$

On the other hand

$$
\frac{1}{4 \pi} \int_{\sigma}\left|P_{n}\left(\cos \psi_{x y}\right)\right| d \sigma_{x} \leq\left(\frac{1}{4 \pi} \int_{\sigma} P_{n}^{2}\left(\cos \psi_{x y}\right) d \sigma_{y}\right)^{1 / 2}=\frac{1}{\sqrt{2 n+1}}
$$

Thus,

$$
M_{N}(r) \leq \mu \sum_{n=N+1}^{\infty} \frac{1}{\sqrt{2 n+1}} \int_{\sigma} G_{n}\left(r, R_{\sigma_{y}}\right) d \sigma_{y},
$$

where $G_{n}\left(r, R_{\sigma_{y}}\right)$ is given by Eq. (B6) with $R=R_{\sigma_{y}}$. The computation of Eq. (31) is somewhat complicated in that one has to split the unit sphere $\sigma$ into two parts

$$
\sigma=\sigma_{+}(r) \cup \sigma_{-}(r), \quad \sigma_{+}(r)=\left\{\sigma: R_{\sigma} \geq r\right\}, \quad \sigma_{-}(r)=\left\{\sigma: R_{\sigma}<r\right\},
$$

and then split the integral of $G_{n}$, using in each part the proper expression of $G_{n}\left(r, R_{\sigma_{y}}\right)$.

This is possible, yet in order to proceed with our analytical estimation, we can consider the two extreme cases $r=R_{0}$ and $r=\bar{R}$, which imply simplified expressions, see Eqs (B8) and (B9). In this case it is convenient to introduce the following definitions

$$
q_{0}=\frac{R_{0}}{\bar{R}}, \quad q_{\sigma}=\frac{R_{\sigma}}{\bar{R}}=\frac{R_{0}+H_{\sigma}}{\bar{R}}=q_{0}+\frac{H_{\sigma}}{\bar{R}} .
$$

Therefore from the Appendix B we find

$$
\begin{aligned}
& M_{N}\left(R_{0}\right) \leq \mu R_{0}^{2} \sum_{n=N+1}^{\infty} \frac{1}{(n-2) \sqrt{2 n+1}} \int_{\sigma}\left[1-\left(\frac{q_{0}}{q_{\sigma}}\right)^{n-2}\right] d \sigma \\
& =\mu R_{0}^{2} \sum_{n=N+1}^{\infty} \frac{1}{(n-2) \sqrt{2 n+1}} \int_{\sigma}\left[1-\left(\frac{1}{1+\frac{H_{\sigma}}{R_{0}}}\right)^{n-2}\right] d \sigma,
\end{aligned}
$$

and 


$$
\begin{aligned}
& M_{N}(\bar{R}) \leq \mu \bar{R}^{2} \sum_{n=N+1}^{\infty} \frac{1}{(n+3) \sqrt{2 n+1}} \int_{\sigma}\left[q_{\sigma}^{n+3}-q_{0}^{n+3}\right] d \sigma \\
& =\mu \bar{R}^{2} \sum_{n=N+1}^{\infty} \frac{1}{(n-2) \sqrt{2 n+1}} \int_{\sigma}\left[\left(q_{0}+\frac{H_{\sigma}}{\bar{R}}\right)^{n+3}-q_{0}^{n+3}\right] d \sigma .
\end{aligned}
$$

We will see in Section 6 an easy numerical estimate of Eqs (33) and (34) for the case of the terrain effect of the Earth, in terms of height anomalies. The figures so derived show that in particular the expression of $M_{N}(\bar{R})$ can be to some extent also of a practical use.

\section{THE MAJORIZATION OF THE MEAN SQUARE ERROR IN THE LAYER $R_{0} \leq r \leq \bar{R}$}

We now repeat the same reasoning of Section 4, but this time to compute the mean square error on the sphere of radius $r$

$$
\mathscr{E}_{N}(\boldsymbol{x})=\left(\frac{1}{4 \pi} \int_{\sigma} \varepsilon_{N}^{2}\left(r, \sigma_{x}\right) d \sigma_{x}\right)^{1 / 2} .
$$

We start again from Eq (12) and at first we apply the Schwarz inequality, getting

$$
\varepsilon_{N}^{2}\left(r, \sigma_{x}\right) \leq \mu^{2} m(\delta B) \int_{\delta B}\left(\sum_{n=N+1}^{\infty} F_{n}(r, s) P_{n}\left(\cos \psi_{x y}\right)\right)^{2} d \boldsymbol{y} .
$$

We integrate Eq. (36) in $d \sigma_{x}$ and, recalling Eq. (19), we obtain

$$
\begin{gathered}
\mathscr{E}_{N}^{2}(r) \leq \mu^{2} m(\delta B) \int_{\delta B}\left[\frac{1}{4 \pi} \int_{\sigma}\left(\sum_{n=N+1}^{\infty} F_{n}(r, s) P_{n}\left(\cos \psi_{x y}\right)\right)^{2} d \sigma_{x}\right] d \boldsymbol{y} \\
=\mu^{2} m(\delta B) \int_{\delta B}\left(\sum_{n=N+1}^{\infty} \frac{F_{n}^{2}(r, s)}{2 n+1}\right) d \boldsymbol{y} .
\end{gathered}
$$

By using the definition of $H_{n}(r, s)$ in Eq. (A7) and expressing the integral of Eq. (37) in spherical coordinates, we arrive at

$$
\mathscr{E}_{N}^{2}(r) \leq \mu^{2} m(\delta B) \sum_{n=N+1}^{\infty} \frac{1}{2 n+1} \int_{\sigma} d \sigma_{y} H_{n}\left(r, R_{\sigma_{y}}\right) .
$$


Repeating the reasoning of Section 4, we give the explicit form of $\mathscr{E}_{N}(r)$ only for the two values $r=R_{0}$ and $r=\bar{R}$, corresponding to the worst and best cases for $\mathscr{E}_{N}$, respectively, and having a more easily computable expression, as we will see in Section 6 . So, recalling Eq. (B14), we find

$$
\begin{aligned}
& \mathscr{E}_{N}^{2}\left(R_{0}\right) \leq \mu \sqrt{m(\delta B)}\left\{\sum_{n=N+1}^{\infty} \frac{R_{0}}{(2 n+1)(2 n-1)} \int_{\sigma}\left[1-\left(\frac{q_{0}}{q_{\sigma_{y}}}\right)^{2 n+3}\right] d \sigma_{y}\right\}^{1 / 2} \\
& =\mu \sqrt{m(\delta B) R_{0}}\left\{\sum _ { n = N + 1 } ^ { \infty } \frac { R _ { 0 } } { ( 2 n + 1 ) ( 2 n - 1 ) } \int _ { \sigma } \left[1-\left(\frac{1}{\left.\left.\left.1+\frac{H_{\sigma_{y}}}{R_{0}}\right)^{2 n+3}\right] d \sigma_{y}\right\}^{1 / 2},}\right.\right.\right.
\end{aligned}
$$

and, recalling Eq. (B13),

$$
\begin{aligned}
& \mathscr{E}_{N}^{2}(\bar{R}) \leq \mu \sqrt{m(\delta B)}\left\{\sum_{n=N+1}^{\infty} \frac{\bar{R}}{(2 n+1)(2 n+3)} \int_{\sigma}\left[q_{\sigma_{y}}^{2 n+3}-q_{0}^{2 n+3}\right] d \sigma_{y}\right\}^{1 / 2} \\
= & \mu \sqrt{m(\delta B) \bar{R}}\left\{\sum_{n=N+1}^{\infty} \frac{\bar{R}}{(2 n+1)(2 n+3)} \int_{\sigma}\left[\left(q_{0}+\frac{H_{\sigma_{y}}}{R_{0}}\right)^{2 n+3}-q_{0}^{2 n+3}\right] d \sigma_{y}\right\}^{1 / 2} .
\end{aligned}
$$

\section{AN APPLICATION OF ERROR BOUNDS TO THE TERRAIN EFFECT OF THE EARTH POTENTIAL}

In this section we will apply formulas of Sections 3-5 to the case of a body constituted by continental masses of the Earth, i.e. those having height $H_{\sigma}>0$ over the geoid, that here is approximated by a sphere of radius $R_{0}$. This is to assess the possible usefulness of the formulas, beyond their theoretical value.

The first question one might pose is whether the majorizations elaborated do apply to a strange body, like that of the topographic masses alone, that in any way covers only about $1 / 3$ of the total sphere of radius $R_{0}$. The answer is yes, because we have essentially derived our formulas starting from the error form given by Eq. (12) which ignores what happens inside $B_{0}$ under condition that the masses there produce the same effect on $u(x)$ and $u_{N}(x)$. This is certainly true because considering the terrain effect as an isolated component of the gravitational potential is the same as putting density $\rho=0$ inside $B_{0}$.

Another doubt one might have is whether the approximation of the geoid with a simple sphere is adequate to the present discussion. Also in this case the answer to the question is in the affirmative sense, because here we are interested only in the majorization of the 
error for a maximum degree $N$ up to the order of several thousands, as it is the computed effect in the spectral form in these years (Balmino et al., 2012); at this high degree, only estimates of the error in the range of centimetres are useful, and a correction to such figures smaller by two orders of magnitude is irrelevant.

In the examples below, we will make use of the ETOPO1 height dataset, with a resolution of $1^{\prime} \times 1^{\prime}$; only $H_{\sigma}>0$ will be considered, i.e. we do not go to the item of "terrain correction" for the oceanic part.

This dataset will be used in a purely statistical sense, to compute integrals of functions $g\left(H_{\sigma}\right)$ over $\sigma$, as explained in Appendix C. As shown in that appendix, the statistical distribution of $H_{\sigma}$, obtained by interpolating the empirical histogram of heights is enough well described by the following probability density function

$$
f(H)=2 \mathrm{e}^{-2 H},
$$

where $H$ is expressed in $\mathrm{km}$; the range of $H$ should go from 0 to $\bar{H}=8 \mathrm{~km}$, which is approximately the maximum height in ETOPO1.

Rigorously this would require dividing by the normalization factor by $1-\mathrm{e}^{-16} \cong$ $\cong 1-10^{-7}$; we will omit this in view of our aim. So, when we want to compute the integral on $\sigma$ of a function $g\left(H_{\sigma}\right)$, with $g(0)=0$, we will borrow on Appendix $\mathrm{C}$ the (approximate) formula:

$$
\int_{\sigma} g\left(H_{\sigma}\right) d \sigma=\int_{\sigma_{L}} g\left(H_{\sigma}\right) d \sigma \cong\left|\sigma_{L}\right| \int_{0}^{\bar{H}} g(H) f(H) \mathrm{d} H,
$$

where $\sigma_{L}$ is the part of the unit sphere where $H_{\sigma}>0$ ( $L$ stands here for land), $\left|\sigma_{L}\right|$ is the area of $\sigma_{L}$ on the unit sphere, i.e.

$$
\left|\sigma_{L}\right| \cong \frac{4 \pi}{3}
$$

and $f(H)$ is given by Eq. (41).

Another warning is that we will assume the constant density of the topography to be $\rho=2.67 \mathrm{~g} \mathrm{~cm}^{-3}$, observing also that this value is about one half of the mean density of the whole Earth, $\rho_{E}$, namely, roughly

so that

$$
\rho_{E} \cong 5.51 \mathrm{~g} \mathrm{~cm}^{-3}, \quad \rho \cong 2.67 \mathrm{~g} \mathrm{~cm}^{-3},
$$

$$
\rho \cong 0.4846 \rho_{E} \cong 0.5 \rho_{E}
$$

Furthermore we will take

$$
R_{0}=6372 \mathrm{~km}, \quad \bar{R}=6380 \mathrm{~km} .
$$




\subsection{Example 1}

We want to compute $C_{N}$ from Eq. (25), for $N=6000,8000,10000$. We set

$$
\begin{aligned}
& m(\delta B)=\int_{\sigma} d \sigma \int_{R_{0}}^{R_{\sigma}} s^{2} \mathrm{~d} s=\frac{1}{3} \int_{\sigma} d \sigma\left(R_{\sigma}^{3}-R_{0}^{3}\right) \\
& \leq \bar{R}^{2} \int_{\sigma} d \sigma H_{\sigma}=\bar{R}^{2} \frac{4}{3} \pi \int_{0}^{\bar{H}} H 2 \mathrm{e}^{-2 H} \mathrm{~d} H \cong \frac{4}{3} \pi \bar{R}^{2} \tilde{H},
\end{aligned}
$$

with $\tilde{H} \cong 0.5 \mathrm{~km}$, and

$$
\mu \bar{R}=G \rho \bar{R}=\frac{1}{2} G \rho_{E} \bar{R}=\frac{1}{2} \frac{G M_{E}}{\frac{4}{3} \pi R_{0}^{3}} \bar{R} \cong \frac{3}{8 \pi} \gamma,
$$

where, in the present approximation, we have put $\bar{R} / R_{0} \cong 1$ and $\gamma=G M_{E} / R_{0}^{2}$.

So we have, by using Eqs (45) and (46),

$$
C_{N}=\mu \sqrt{\frac{1}{3} \frac{(4 \pi)^{2} \bar{R}^{3} \tilde{H}}{2(N+1)}}=\mu \bar{R}^{2} \frac{4 \pi}{\sqrt{6}} \sqrt{\frac{\tilde{H} / \bar{R}}{N+1}} \cong \frac{\sqrt{3}}{2 \sqrt{2}} \bar{R} \sqrt{\frac{\tilde{H} / \bar{R}}{N+1}} \gamma .
$$

With this we computed the results listed in Table 1.

Let us notice that we have divided our error estimate by $\gamma$, to express it in terms of height anomaly, in order to make it more readable. As anticipated in Section 3, such figures are of no practical use.

\subsection{Example 2}

In this example we compute $M_{N}\left(R_{0}\right)$ and $M_{N}(\bar{R})$ from Eqs (33) and (34). As for Eq. (33), exploiting the averaging formula of Eq. (42), we get

$$
M_{N}\left(R_{0}\right) \leq \mu R_{0}^{2} \sum_{n=N+1}^{\infty} \frac{4 \pi}{3(n-2) \sqrt{2 n+1}} \int_{0}^{\bar{H}}\left\{1-\frac{1}{\left[1+\left(H / R_{0}\right)\right]^{n-2}}\right\} 2 \mathrm{e}^{-2 H} \mathrm{~d} H .
$$

Of course one could make a more precise calculation of Eq. (48), yet since the integrals there tend monotonously to 1 for $n \rightarrow \infty$ and are of the order of $0.6-0.7$ when $n$ ranges

Table 1. Upper bound of the error in terms of height anomaly computed by Eq. (47).

\begin{tabular}{|r|c|}
\hline$N$ & $C_{N} / \gamma[\mathrm{m}]$ \\
\hline 6000 & 447 \\
8000 & 387 \\
10000 & 346 \\
\hline
\end{tabular}




\section{Approximation of the potential of a homogeneous almost spherical body}

between 6000 and 10000, it is enough here to compute the order of magnitude of the constants

$$
K_{N}=\mu R_{0}^{2} \frac{4}{3} \pi \sum_{n=N+1}^{\infty} \frac{1}{(n-2) \sqrt{2 n+1}}
$$

with $\mu R_{0} \cong 3 \gamma / 8 \pi$, given by Eq. (46), and, with the approximation

$$
\sum_{n=N+1}^{\infty} \frac{1}{(n-2) \sqrt{2 n+1}} \cong \frac{1}{\sqrt{2}} \int_{N+1}^{\infty} \frac{d x}{x^{3 / 2}}=\frac{\sqrt{2}}{\sqrt{N+2}},
$$

we see that

$$
K_{N} \cong \frac{\gamma}{2} R_{0} \frac{\sqrt{2}}{\sqrt{N+1}},
$$

so that $K_{N} / \gamma$ is more than $10 \mathrm{~km}$ and its possible usefulness is null.

Different is the story for $M(\bar{R})$, which, according to Eqs (A13) and (34), we write as

$$
M_{N}(\bar{R}) \leq h_{N}=\mu \bar{R}^{2} \sum_{n=N+1}^{\infty} \frac{1}{(n+3) \sqrt{2 n+1}} \int_{\sigma}\left[\left(q_{0}+\frac{H_{\sigma}}{\bar{R}}\right)^{n+3}-q_{0}^{n+3}\right] d \sigma .
$$

Exploiting again Eq. (42), we find, noting that the integrand goes to zero when $H_{\sigma}=0$,

$$
\int_{\sigma_{L}}\left[\left(q_{0}+\frac{H_{\sigma}}{\bar{R}}\right)^{n+3}-q_{0}^{n+3}\right] d \sigma \cong \frac{4 \pi}{3} \int_{0}^{\bar{H}}\left[\left(q_{0}+\frac{H}{\bar{R}}\right)^{n+3}-q_{0}^{n+3}\right] 2 \mathrm{e}^{-2 H} \mathrm{~d} H .
$$

The integrals in Eq. (52) can be computed numerically by Eq. (53) and we report the result of $M_{N} / \gamma$, providing the majorization in terms of height anomaly. Recalling Eqs (46) and (50) and noting that the integral in Eq. (53) decreases with $N$, when $n>6000$, we find

$$
\frac{M_{N}(\bar{R})}{\gamma} \leq \frac{h_{N}}{\gamma} \leq \bar{R}\left\{\int_{0}^{\bar{H}}\left[\left(q_{0}+\frac{H}{\bar{R}}\right)^{N+4}-q_{0}^{N+4}\right] \mathrm{e}^{-2 H} \mathrm{~d} H\right\} \frac{\sqrt{2}}{\sqrt{N+1}},
$$

leading to the results reported in Table 2 .

As we can see the range of the bounds of the mean absolute value on the sphere of radius $\bar{R}$, although rather large, starts to be reasonable in comparison to the ones of

Table 2. Upper bound of the error in terms of height anomaly computed by Eq. (54).

\begin{tabular}{|r|c|}
\hline$N$ & $h_{N} / \gamma[\mathrm{m}]$ \\
\hline 6000 & 55.6 \\
8000 & 7.4 \\
10000 & 1.2 \\
\hline
\end{tabular}




\section{B. Bucha et al.}

Table 1, computed for Example 1. In any event they are quite far, for instance, from estimates of the error of recent EGM models.

$$
\text { 6.3. Example } 3
$$

In this example we compute the bounds for $\mathscr{E}_{N}\left(R_{0}\right) / \gamma$ and $\mathscr{E}_{N}(\bar{R}) / \gamma$ from Eqs (39) and (40), respectively. We proceed as in Example 2 and we write first

$$
\begin{gathered}
\mathscr{E}_{N}\left(R_{0}\right) \leq \mu \sqrt{m(\delta B) R_{0}} \\
\times\left\{\sum_{n=N+1}^{\infty} \frac{4 \pi}{3} \frac{1}{(2 n+1)(2 n-1)} \int_{0}^{\bar{H}}\left[1-\frac{1}{\left(1+\frac{H}{R_{0}}\right)^{2 n+3}}\right] 2 \mathrm{e}^{-2 H} \mathrm{~d} H\right\}^{1 / 2} .
\end{gathered}
$$

Observing that for $n \rightarrow \infty$ the integrals in Eq. (55) tend to 1 from below, we can only majorize $\mathscr{E}_{N}\left(R_{0}\right)$ as

$$
\begin{aligned}
\mathscr{E}_{N}\left(R_{0}\right) & \leq \mu \sqrt{m(\delta B) R_{0} \frac{4 \pi}{3}} \sqrt{\sum_{n=N+1}^{\infty} \frac{1}{(2 n+1)(2 n-1)}} \\
& =\mu \sqrt{m(\delta B) R_{0} \frac{4 \pi}{3}} \frac{1}{\sqrt{2(2 N+1)}} .
\end{aligned}
$$

So, recalling that (see Eqs (45) and (46))

$$
\begin{gathered}
\sqrt{m(\delta B)}=\sqrt{\frac{4 \pi}{3} R_{0}^{2} \tilde{H}}, \quad \tilde{H}=0.5 \mathrm{~km}, \\
\mu R_{0}=\frac{3}{8 \pi} \gamma,
\end{gathered}
$$

we finally find

$$
\frac{\mathscr{E}_{N}\left(R_{0}\right)}{\gamma} \leq \frac{R_{0}}{2} \sqrt{\frac{\tilde{H} / R_{0}}{4 N+2}}=\frac{j_{N}}{\gamma},
$$

yielding results listed in Table 3.

Once again we see that for $r=R_{0}$ our majorizations are of little practical insight. On the contrary when we fix $r=\bar{R}$, we have

Table 3. Upper bound of the error in terms of height anomaly computed by Eq. (59).

\begin{tabular}{|r|c|}
\hline$N$ & $j_{N} / \gamma[\mathrm{m}]$ \\
\hline 6000 & 182 \\
8000 & 157 \\
10000 & 141 \\
\hline
\end{tabular}




$$
\begin{gathered}
\mathscr{E}_{N}(\bar{R}) \leq \mu \sqrt{m(\delta B) \bar{R}} \\
\times\left\{\sum_{n=N+1}^{\infty} \frac{4 \pi}{3} \frac{1}{(2 n+1)(2 n+3)} \int_{0}^{\bar{H}}\left[\left(q_{0}+\frac{H}{\bar{R}}\right)^{2 n+3}-q_{0}^{2 n+3}\right] 2 \mathrm{e}^{-2 H} \mathrm{~d} H\right\}^{1 / 2} .
\end{gathered}
$$

A quick numerical evaluation of the integrals in Eq. (60) shows that they are decreasing for $n$ in the the range we are interested in, so Eq. (60) can be written in the form

$$
\begin{gathered}
\mathscr{E}_{N}(\bar{R}) \leq \mu \sqrt{m(\delta B) \bar{R} \frac{4 \pi}{3}} \\
\times\left\{\int_{0}^{\bar{H}}\left[\left(q_{0}+\frac{H}{\bar{R}}\right)^{2 N+5}-q_{0}^{2 N+5}\right] 2 \mathrm{e}^{-2 H} \mathrm{~d} H \sum_{n=N+1}^{\infty} \frac{1}{(2 n+1)(2 n+3)}\right\}^{1 / 2} .
\end{gathered}
$$

With the identity

$$
\sum_{n=N+1}^{\infty} \frac{1}{(2 n+1)(2 n+3)}=\frac{1}{2} \sum_{n=N+1}^{\infty}\left(\frac{1}{2 n+1}-\frac{1}{2 n+3}\right)=\frac{1}{4 N+6},
$$

and recalling Eqs (57) and (58), we arrive at the formula

$$
\mathscr{E}_{N}(\bar{R}) \leq \gamma \frac{\bar{R}}{2} \sqrt{\frac{\tilde{H} / \bar{R}}{4 N+6}}\left\{\int_{0}^{\bar{H}}\left[\left(q_{0}+\frac{H}{\bar{R}}\right)^{2 N+5}-q_{0}{ }^{2 N+5}\right] 2 \mathrm{e}^{-2 H} \mathrm{~d} H\right\}^{1 / 2}=\ell_{N} .
$$

Results of direct numerical evaluation of Eq. (63) are listed in Table 4.

As we can see these figures, although derived from simple majorizations, seem quite reasonable and might be useful for a quick assessment of spectral calculations.

Table 4. Upper bound of the error in terms of height anomaly computed by Eq. (63).

\begin{tabular}{|r|c|}
\hline$N$ & $\ell_{N} / \gamma[\mathrm{mm}]$ \\
\hline 6000 & 83.9 \\
8000 & 29.1 \\
10000 & 17.6 \\
\hline
\end{tabular}




\section{B. Bucha et al.}

\section{CONCLUSIONS}

After discussing the various types of spectral approximations of the gravitational potential of the body $B$, we focused on the so called internal-external approach, by examining its error in the topographic layer, i.e. for $\boldsymbol{x} \in \bar{B} \backslash B_{0} \equiv\left\{R_{0} \leq r \leq \bar{R}\right\}$, including the volume occupied by masses.

Three types of norms are used to control the error $\varepsilon_{N}(\boldsymbol{x})$, namely $C\left(\bar{B} \backslash B_{0}\right)$, the $L_{1}(\sigma)$ norm, also called mean absolute value of $\varepsilon_{N}$ on a sphere contained in $\bar{B} \backslash B_{0}$, and the $L_{2}(\sigma)$ norm, also called mean square error on a sphere in $\bar{B} \backslash B_{0}$ (see Sections 3,4 , and 5 , respectively). The formulas so derived are then applied to the case of the Earth terrain effect and evaluated, in particular for the $L_{1}$ and $L_{2}$ instances, fixing $r$ to its extreme values, namely $r=R_{0}$ and $r=\bar{R}$.

The conclusions are as follows:

- The norm in $C\left(\bar{B} \backslash B_{0}\right)$ of the error goes slowly to zero, proving the convergence of the method even within masses, but the application to the potential of the Earth topographic masses shows that the majorization is too rough to be useful.

- The convergence of the mean absolute and the mean square error on the maximal Bjerhammar sphere (i.e. $r=R_{0}$ ) is also guaranteed, but its numerical evaluation for the example of the Earth terrain effect shows that majorizations are useless on a practical ground.

- On the contrary the evaluation of the mean absolute and the mean square errors on the minimal Brillouin sphere for the Earth example, shows that, specially for the $L_{2}$ case, the majorizations could be usefully applied also to practical problems.

Summarizing, we deem that beyond a certain theoretical value and a possible application to complicated bodies like asteroids, the examples on Earth terrain correction deserve some consideration, at least for the mean square error at very high degrees and various altitudes. In fact, one has to consider that, e.g. at degree 10000 , corresponding roughly to $2-\mathrm{km}$ resolution at ground, the spectral omission error of the topographic terrain masses is likely to be almost equal to the omission error of the whole gravity potential, implying that an upper bound of the former is approximately valid for the latter too. Therefore, knowing that the omission error of a gravity model with a maximum degree of 10000 cannot be larger than $1.7 \mathrm{~cm}$ is a valuable information itself. Once more, to interpret correctly such a figure, one has to recall that this is only the upper bound, which is certainly pessimistic, as it is in the nature of mathematical majorization.

Finally, the calculation of the spectral error in terms of gravity anomaly in the topographic layer is still under study and might be a subject of a further paper. 


\section{APPENDIX A}

Here we briefly justify the steps from Eq. (12) to Eq. (20). The Schwarz inequality claims that given any measurable set $C$ in $\mathbb{R}^{n}$ (in our case $n=3$ ) and two functions $f(y)$, $g(y)$ square integrable over $C$, one has

$$
\left|\int_{C} f(y) g(y) d C\right|^{2} \leq \int_{C} f^{2}(y) d C \int_{C} g^{2}(y) d C .
$$

Equation (A1) holds for any $f(y), g(y) \in L_{2}(C)$, and it becomes an equality if and only if $f(y)$ is proportional to $g(y)$ (Sansò and Sideris, 2012). Hence, if we use

$$
f(y)=1, \quad g(y)=\frac{1}{\ell_{x y}}-\frac{1}{\ell_{N, x y}},
$$

for a fixed $x$ in Eq. (12) and using Eq. (A1) we obtain Eq. (20). Yet this is valid only if both $f(y), g(y)$ are square integrable on $C$. For $f=1$ this is true if $C$ is a bounded set, as it is in our case, so that

$$
\int_{C}^{1} 1^{2} d C=\mu(C) .
$$

So the question is whether, with $C$ bounded,

$$
\left(\frac{1}{\ell_{x y}}-\frac{1}{\ell_{N, x y}}\right) \in L_{2}(C) .
$$

Now, $1 / \ell_{N, x y}$ is continuous and therefore bounded if the origin is outside $C$, which is the case when we take $C=\delta B$. So, $1 / \ell_{N, x y} \in L_{2}$. Moreover, we claim that, for any fixed $x$ and bounded $C$,

$$
I=\int_{C} \frac{1}{\ell_{x y}^{2}} d C<+\infty .
$$

In fact, if we use a spherical coordinate system with the origin in $x$, we have

$$
I=\int_{C} \frac{1}{r^{2}} r^{2} d r d \sigma .
$$


Since $C$ is bounded, it is contained in a sphere $B_{\bar{R}}$ for some large radius $\bar{R}$, so that

$$
I \leq \int_{B_{\bar{R}}} \frac{1}{r^{2}} r^{2} d r d \sigma=\int d \sigma \int_{0}^{\bar{R}} \mathrm{~d} r=4 \pi \bar{R}<+\infty
$$

which we wanted to prove. Finally, since both $1 / \ell_{x y}$ and $1 / \ell_{N}, x y$ are in $L^{2}(C)$, the same is true for $\left(1 / \ell_{x y}\right)-\left(1 / \ell_{N}, x y\right)$. Therefore, Eq. (20) is justified.

\section{APPENDIX B}

In this Appendix we report the calculation of the integrals

$$
\begin{aligned}
& G_{n}(r, R)=\int_{R_{0}}^{R} F_{n}(r, s) s^{2} \mathrm{~d} s, \\
& H_{n}(r, R)=\int_{R_{0}}^{R} F_{n}^{2}(r, s) s^{2} \mathrm{~d} s,
\end{aligned}
$$

where $r$ and $R$ can attain any value between $R_{0}$ and $\bar{R}$. Since we need $G_{n}$ and $H_{n}$ also when $R<\bar{R}$, we can have for such functions two distinct expressions, according whether $r \geq R$ or $r<R$. From Eq. (5) we have, for $n>2$,

$$
G_{n}(r, R)=\int_{R_{0}}^{R}\left[\frac{s^{n+2}}{r^{n+1}} H(r-s)+\frac{r^{n}}{s^{n-1}} H(s-r)\right] \mathrm{d} s .
$$

Now, for $r<R$ one gets, also recalling the definition of the Heaviside function $H(t)$,

$$
\begin{gathered}
G_{n}(r, R)=\int_{R_{0}}^{r} \frac{s^{n+2}}{r^{n+1}} H(r-s) \mathrm{d} s+\int_{r}^{R} \frac{r^{n}}{s^{n-1}} H(s-r) \mathrm{d} s \\
=\frac{1}{2+3}\left(r^{2}-\frac{R_{0}^{n+3}}{r^{n+1}}\right)-\frac{1}{n-2}\left(\frac{r^{n}}{R^{n-2}}-r^{2}\right) \\
=r^{2}\left\{\frac{1}{n+3}\left[1-\left(\frac{R_{0}}{r}\right)^{n+3}\right]+\frac{1}{n-2}\left[a-\left(\frac{r}{R}\right)^{n-2}\right]\right\} .
\end{gathered}
$$

On the contrary, when $r>R$, Eq. (B3) yields

$$
G_{n}(r, R)=\int_{R_{0}}^{R} \frac{s^{n+2}}{r^{n+1}} \mathrm{~d} s=\frac{1}{n+3}\left(\frac{R^{n+3}}{r^{n+1}}-\frac{R_{0}^{n+3}}{r^{n+1}}\right)=r^{2} \frac{1}{n+3}\left(\frac{R}{r}\right)^{n+3}\left[1-\left(\frac{R_{0}}{R}\right)^{n+3}\right]
$$


Equations (B4) and (B5) can be summarized in a compound formula as

$$
\begin{aligned}
G_{n}(r, R)=r^{2} & \left\{\left[\frac{1}{n+3}\left(1-\left(\frac{R_{0}}{r}\right)^{n+3}\right)+\frac{1}{n-2}\left(1-\left(\frac{r}{R}\right)^{n-2}\right)\right] H(R-r)\right. \\
& \left.+\frac{1}{n+3}\left(\frac{R}{r}\right)^{n+3}\left[1-\left(\frac{R_{0}}{R}\right)^{n+3}\right] H(r-R)\right\} .
\end{aligned}
$$

Of course we are going to use Eq. (B6) for large values of $n$, so we are not interested in $G_{0}$ and $G_{1}$. Particular values of $R$ in Eq. (B6) give

$$
\begin{gathered}
G_{n}(r, \bar{R})=r^{2}\left\{\frac{1}{n+3}\left[1-\left(\frac{R_{0}}{r}\right)^{n+3}\right]+\frac{1}{n-2}\left[1-\left(\frac{r}{\bar{R}}\right)^{n-2}\right]\right\} \\
=r^{2}\left[c_{n}-\frac{1}{n+3}\left(\frac{R_{0}}{r}\right)^{n+3}-\frac{1}{n-2}\left(\frac{r}{\bar{R}}\right)^{n-2}\right], \quad 0<r \leq \bar{R},
\end{gathered}
$$

where $c_{n}=\frac{2 n+1}{(n+3)(n-2)}$,

$$
G_{n}\left(\bar{R}, R_{\sigma}\right)=\frac{\bar{R}^{2}}{n+3}\left(\frac{R_{\sigma}}{\bar{R}}\right)^{n+3}\left[1-\left(\frac{R_{0}}{R_{\sigma}}\right)^{n+3}\right]=\frac{\bar{R}^{2}}{n+3}\left(q_{\sigma}^{n+3}-q_{0}^{n+3}\right)
$$

where we have used the definition of Eq (32), $q_{0}=R_{0} / \bar{R}, q_{\sigma}=R_{\sigma} / \bar{R}$,

$$
G_{n}\left(R_{0}, R_{\sigma}\right)=\frac{R_{0}^{2}}{n-2}\left[1-\left(\frac{R_{0}}{R_{\sigma}}\right)^{n-2}\right]=\frac{R_{0}^{2}}{n-2}\left[1-\left(\frac{q_{0}}{q_{\sigma}}\right)^{n-2}\right] .
$$

In particular, form Eq. (B9), it follows

$$
G_{n}(r, \bar{R}) \leq c_{n} r^{2} \leq c_{n} \bar{R}^{2} .
$$

As for Eq. (B2), a similar computation can be conducted, in this case for $n>0$. To perform this, it is convenient to use the relations, valid for every $t \neq 0$,

$$
H^{2}(t)=H(t), \quad H(t) H(-t)=0 .
$$


The results is

$$
\begin{gathered}
H_{n}(r, R)=r\left\{\left[\frac{1}{2 n+3}\left(1-\left(\frac{R_{0}}{r}\right)^{2 n+3}\right)+\frac{1}{2 n-1}\left(1-\left(\frac{r}{R}\right)^{2 n-1}\right]\right] H(R-r)\right. \\
\left.+\frac{1}{2 n+3}\left(\frac{R}{r}\right)^{2 n+3}\left(1-\left(\frac{R_{0}}{R}\right)^{2 n+3}\right) H(r-R)\right\} \\
=r\left\{\left[a_{n}-\frac{1}{2 n+3}\left(\frac{R_{0}}{r}\right)^{2 n+3}-\frac{1}{2 n-1}\left(1-\left(\frac{r}{R}\right)^{2 n 31}\right)\right] H(R-r)\right] \\
\left.+\frac{1}{2 n+3}\left(\frac{R}{r}\right)^{2 n+3}\left(1-\left(\frac{R_{0}}{R}\right)^{2 n+3}\right) H(r-R)\right\},
\end{gathered}
$$

where $a_{n}=\frac{2(2 n+1)}{(2 n+3)(2 n-1)}$.

Useful particular values of $R$ in Eq. (B12) give

$$
\begin{gathered}
H_{n}\left(\bar{R}, R_{\sigma}\right)=\frac{\bar{R}}{2 n+3}\left(\frac{R_{\sigma}}{\bar{R}}\right)^{2 n+3}\left[1-\left(\frac{R_{0}}{R_{\sigma}}\right)^{2 n+3}\right]=\frac{\bar{R}}{2 n+3}\left(q_{\sigma}^{2 n+3}+q_{0}^{2 n+3}\right), \\
H_{n}\left(R_{0}, R_{\sigma}\right)=\frac{R_{0}}{2 n-1}\left[1-\left(\frac{R_{0}}{R_{\sigma}}\right)^{2 n+3}\right]=\frac{R_{0}}{2 n-1}\left[1-\left(\frac{q_{0}}{q_{\sigma}}\right)^{2 n+3}\right] .
\end{gathered}
$$

Moreover, from Eq.(B12), considering that $a_{n}>1 /(2 n+3)$ for every $n \geq 0$, one also has

$$
\forall R \leq \bar{R}, \quad H_{n}(r, R) \leq a_{n} r \leq a_{n} \bar{R} .
$$

\section{APPENDIX C}

The aim of this Appendix is to find an approximate formula to compute

$$
I=\int_{A} g\left(H_{\sigma}\right) d \sigma,
$$

when $g\left(H_{\sigma}\right)$ is any integrable function on the unit sphere $\sigma$, sampled at a regular geographical grid of points $P_{i k} \equiv\{i \Delta \vartheta, k \Delta \lambda\} \in A$. To achieve our aim, the focus here, rather than on a quadrature formula, implying the use of each individual value 
$g_{i k}=g\left(H_{P_{i k}}\right)$, is on using the histograms of the values $\left\{H_{P_{i k}}\right\}$, that in the case of the dataset ETOPO1, restricted to the land part of the globus, is readily available (see Fig. C1). As we see from this figure, a "good" interpolation of the histogram by a probability density function is

$$
\tilde{f}(H)=C \mathrm{e}^{-2 H}, \quad 0 \leq H \leq \bar{H}=8 \mathrm{~km},
$$

where

$$
C=2\left(1-\mathrm{e}^{-16}\right)^{-1} \cong 2
$$

We note already here that the average height of this distribution is

$$
\tilde{H}=0.5 \mathrm{~km},
$$

a value often used in Section 6.

Going back to our aim, we will use the mapping of the unit sphere $\sigma$ on the $\mathbb{R}^{2}$ rectangle $\mathbb{R}=\{0 \leq \vartheta \leq \pi, 0 \leq \lambda \leq 2 \pi\}$.
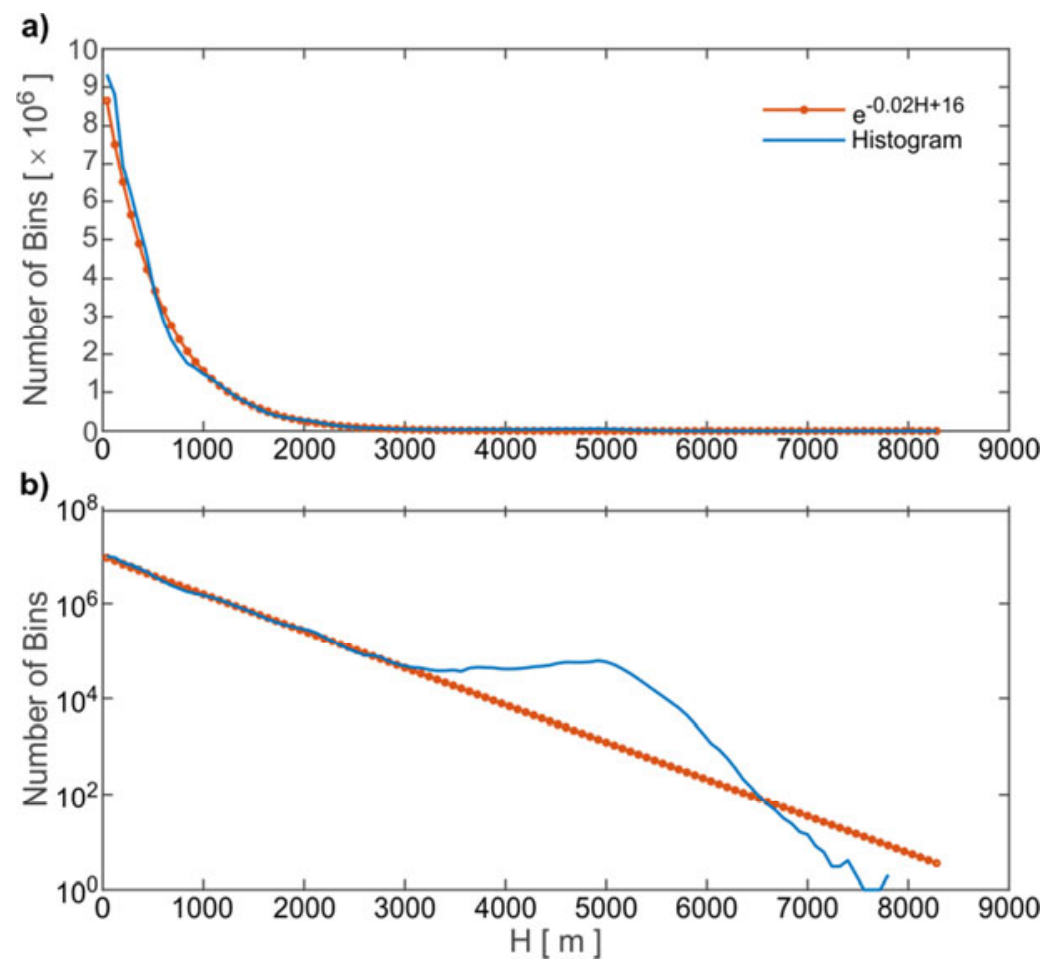

Fig. C1. The histogram of the heights $H$ of ETOPO1 dataset, and its interpolation by an exponential function; a) original, b) logarithmic representation. 


\section{B. Bucha et al.}

Let us notice that the area element $d \sigma$ of the sphere is related to the area element on the map, $d \tilde{\sigma}=d \vartheta d \lambda$ (see Fig. C2), by the equation

$$
d \sigma=\sin \vartheta d \tilde{\sigma} .
$$

Now we have

$$
I=\int_{A} g\left(H_{\sigma}\right) d \sigma=\int_{\tilde{A}} g\left(H_{\sigma}\right) \sin \vartheta d \tilde{\sigma} \cong \tilde{m}(\tilde{A}) \tilde{E}\left[g\left(H_{\sigma}\right)\right] \tilde{E}[\sin \vartheta],
$$

where $\tilde{m}(\tilde{A})$ is the measure of $\tilde{A}$ with the element $d \tilde{\sigma}$. The approximation of the mean of a product with the product of the means is rough, though adequate to the level of accuracy we are aiming at.

Continuing from Eq. (C6), we notice that

$$
\tilde{m}(\tilde{A}) \tilde{E}[\sin \vartheta]=\int_{A} \sin \vartheta d \vartheta d \lambda=m(A),
$$

$m(A)$ being the area of $A$, with respect to the spherical surface element $d \sigma$. In the case of ETOPO1 we will take

$$
m(A)=m\left(\sigma_{L}\right) \cong \frac{4 \pi}{3}
$$

So substituting Eq. (C7) into Eq. (C6) we arrive at

$$
I \cong m(A) \tilde{E}\left[g\left(H_{\sigma}\right)\right]=m(A) \int_{0}^{\bar{H}} g(H) \tilde{f}(H) \mathrm{d} H,
$$

with $\tilde{f}(H)$ given by Eq. (C2) in our case.

Therefore, by accepting the above approximation, Eq. (42) is justified.
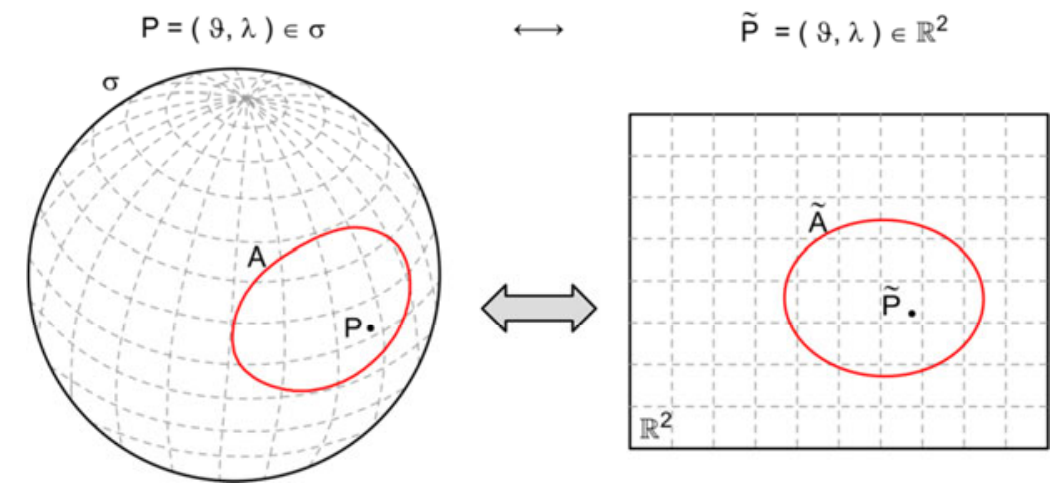

Fig. C2. Mapping of the unit sphere $\sigma$ on the $\mathbb{R}^{2}$ rectangle. 


\section{Approximation of the potential of a homogeneous almost spherical body}

Open Access: This article is distributed under the terms of the Creative Commons Attribution 4.0 International License (http://creativecommons.org/license/by/4.0), which permits use, duplication, distribution and reproduction in any medium or format, as long as you give appropriate credit to the original author(s) and the source, provide a link to the Creative Commons license and indicate if changes were made.

\section{References}

Ågren J., 2004. The analytical continuation bias in geoid determination using potential coefficients and terrestrial gravity data. J. Geodesy, 78, 314-332, DOI: 10.1007/s00190-004-0395-0

Balmino G., 1994. Gravitational potential harmonics from the shape of an homogeneous body. Celest. Mech. Dyn. Astron., 60, 331-364

Balmino G., Vales N., Bonvalot S. and Briais A., 2012. Spherical harmonic modelling to ultra-high degree of Bouguer and isostatic anomalies. J. Geodesy, 86, 499-520, DOI: 10.1007/s00190011-0533-4

Bucha B. and Sansò F., 2021. Gravitational field modelling near irregularly shaped bodies using spherical harmonics: a case study for the asteroid (101955) Bennu. J. Geodesy, 95, Art.No. 56, DOI: $10.1007 / \mathrm{s} 00190-021-01493-\mathrm{w}$

Bucha B., Hirt C. and Kuhn M., 2019. Divergence-free spherical harmonic gravity field modelling based on the Runge-Krarup theorem: a case study for the Moon. J. Geodesy, 93, 489-513, DOI: $10.1007 / \mathrm{s} 00190-018-1177-4$

Freeden W. and Schreiner M., 2009. Spherical Functions of Mathematical Geosciences: A Scalar, Vectorial, and Tensorial Setup. Springer-Verlag, Berlin, Germany

Górski K.M., Bills B.G. and Konopliv A.S., 2018. A high resolution mars surface gravity grid. Planet. Space Sci., 160, 84-106, DOI: 10.1016/j.pss.2018.03.015

Hirt C. and Kuhn M., 2014. Band-limited topographic mass distribution generates full-spectrum gravity field: Gravity forward modeling in the spectral and spatial domains revisited. J. Geophys. Res.-Solid Earth, 119, 3646-3661, DOI: 10.1002/2013JB010900

Hirt C. and Kuhn M., 2017. Convergence and divergence in spherical harmonic series of the gravitational field generated by high-resolution planetary topography - A case study for the Moon. J. Geophys. Res.-Planets, 122, 1727-1746, DOI: 10.1002/2017JE005298

Hirt C., Reußner E., Rexer M. and Kuhn M., 2016. Topographic gravity modeling for global Bouguer maps to degree 2160: Validation of spectral and spatial domain forward modeling techniques at the 10 microGal level. J. Geophys. Res.-Solid Earth, 121, 6846-6862, DOI: $10.1002 / 2016 J B 013249$

Hirt C., Yang M., Kuhn M., Bucha B., Kurzmann A. and Pail R., 2019. SRTM2gravity: an ultrahigh resolution global model of gravimetric terrain corrections. Geophys. Res. Lett., 46, 4618-4627, DOI: 10.1029/2019GL082521

Hotine M., 1969. Mathematical Geodesy. ESSA Monograph 2. U.S. Environmental Science Services Administration, Washington, DC

Jekeli C., 1983. A numerical study of the divergence of spherical harmonic series of the gravity and height anomalies at the Earth's surface. Bull. Geod., 57, 10-28, DOI: 10.1007/BF02520909

Jekeli C., 2017. Spectral Methods in Geodesy and Geophysics. CRC Press, Boca Raton, FL 


\section{B. Bucha et al.}

Martinec Z., 1998. Boundary-Value Problems for Gravimetric Determination of a Precise Geoid. Lecture Notes in Earth Sciences, 73. Springer-Verlag, Berlin, Germany

Moritz H., 1980. Advanced Physical Geodesy. Herbert Wichmann Verlag, Karlsruhe, Germany

Reimond S. and Baur O., 2016. Spheroidal and ellipsoidal harmonic expansions of the gravitational potential of small solar system bodies. Case study: Comet 67P/Churyumov-Gerasimenko. J. Geophys. Res.-Planets, 121, 497-515, DOI: 10.1002/2015JE004965

Sansò F. and Sideris M.G. (Eds), 2012. Geoid Determination: Theory and Methods. Lecture Notes in Earth System Sciences, Springer-Verlag, Berlin, Germany

Sjöberg L.E., 1977. On the Errors of Spherical Harmonic Developments of Gravity at the Surface of the Earth. Report 257. Department of Geodetic Sciences, The Ohio State University, Columbus, $\mathrm{OH}$

Sjöberg L.E., 1999. On the downward continuation error at the Earth's surface and the geoid of satellite derived geopotential models. Boll. Geod. Sci. Affini, 58, 215-229

Takahashi Y. and Scheeres D., 2014. Small body surface gravity fields via spherical harmonic expansions. Celest. Mech. Dyn. Astron., 119, 169-206, DOI: 10.1007/s10569-014-9552-9

Wieczorek M.A., 2015. Gravity and topography of the terrestrial planets. In: Schubert G. and Spohn T. (Eds), Physics of Terrestrial Planets and Moons. Treatise on Geophysics (Second Edition), 10, 153-193, Elsevier, Amsterdam, The Netherlands 